\title{
Control of Hemorrhage in Liver Trauma.
}

\author{
Skënder Buci ${ }^{1}$, Dorina Shtjefni ${ }^{1}$, Arben Gjata ${ }^{2}$, Gjovalin Bushi ${ }^{1}$, Sonja \\ (Saraçi) Butorac ${ }^{3}$ \\ https://doi.org/10.32391/ajtes.v4i1.78
}

\begin{abstract}
Background: Control of hemorrhage in major liver trauma constitutes an ongoing surgical challenge even in nowadays, as surgeons often face difficult situations in its management.

The goal of this study was to analyze our experience in the control of hemorrhage in major liver trauma.

Materials and methods: Our study was performed prospectively covering a period of time from January 2009 till December 2012. 173 patients with liver trauma were included in our study.

Results: Temporary haemostasis maneuvers in the setting of urgent laparotomy consisted of: bimanual compression 13 patients (15.3\%), Pringle maneuver 23 patients $(27 \%)$, perihepatic packing 10 patients $(11,8 \%)$, Foley catheter balloon tamponade 2 patients $(2.4 \%)$, intrahepatic tamponade in penetrating trauma 4 patients $(4.7 \%)$.

Definitive haemostasis methods used in urgent laparotomy were: electro-coagulation of the liver injury 12 patients (14.1\%), haemostasis and biliostasis followed by hepatic closure 41 patients $(48.2 \%)$, haemostasis and biliostasis without hepatic closure 6 patients $(7.1 \%)$, tamponade with a pedicled vascularized omental flap (pedicled omentum hepatorrhaphy) 7 patients $(8.2 \%)$, anatomic liver resection 1 patient $(1.2 \%)$, nonanatomic/atypical liver resection 7 patients $(8.2 \%)$, right hepatic artery ligation 1 patient $(1.2 \%)$.

Surgical techniques in preplanned laparotomies consisted of: left hepatectomy 1 patient $(16.6 \%)$, right hepatectomy 1 patient $(16,6 \%)$, nonanatomic/atypical liver resections 4 patients $(66.7 \%)$. The success of treatment was significantly related to the grade of liver injury $(z=5.2912$, $\mathrm{p}=<0.00001)$, other concomitant abdominal organ injuries $(\mathrm{z}=4.0743, \mathrm{p}=0.00005)$, amount of blood transfusion received $(p=0.03207)$, and age $(p=0.04944)$.

Overall mortality rate was $13 \%$.

Conclusion: Perihepatic packing and performing the surgical intervention in two sessions has significantly improved survival rates in major liver trauma from our experience.

Keywords: liver trauma, haemostasis maneuvers, resection, tamponade.
\end{abstract}

*Corresponding Author: Skënder Buci

$\equiv \bowtie$ E-mail: buciskender@gmail.com

${ }^{1}$ Department of Surgery, University Hospital of Trauma, Tirana, Albania

2 Department of General Surgery, UHC "Mother Teresa", Tirana, Albania.

${ }^{3}$ Department of Radiology, University Hospital of Trauma, Tirana, Albania. 


\section{Full Text}

\section{Introduction}

Hemorrhage in major liver trauma often confronts surgeons with challenging situations in its management. A perfect knowledge of surgical anatomy is therefore crucial in performing an efficient and safe intervention.

Liver surgical anatomy is based on the concept of vascular anatomic division of liver according to Claud Couinaud, Thon That Tung and Henri Bismuth, who divided the liver in independent segments that can each be treated separately.

Management of polytrauma patients ought to be performed by a multidisciplinary in-hospital team to perform a rapid assessment of injuries, to determine the hierarchy of the most urgent priorities and to organize the conditions for efficient treatment.

Timely diagnosis and treatment of these injuries rises survival rates in comparison with patients undergoing delayed treatment.

Abdominal ultrasound is an essential examination in an emergency setting for hemodynamically unstable patients. TDM nowadays constitutes the examination of choice in the evaluation and follow-up of patients with blunt liver trauma who are hemodynamically stable or who are stabilized following transfusion.

Liver injury is encountered in 20\% of polytrauma patients with blunt trauma (1).
Extrahepatic bile duct injuries and injury to the portal triad are encountered in $0,07 \%-0,21 \%$ of all trauma patients admitted in first level trauma centers, with an overall mortality of 50\% (2), (3).

Nearly two decades before, all traumatic liver injuries underwent open surgery, but in 50 to $80 \%$ of cases there was no active bleeding detected (4), (5).

Short-timed laparotomy and surgery performed in two sessions has reduced mortality by $30 \%$ to $70 \%$. (6).

Reported failure of conservative treatment is $15 \%$ and often it is due to extrahepatic causes or negative laparotomy (7), (8).

Success in conservative management of liver injuries are closely related to the ongoing progress in the radiology field.

\section{Aim of the study}

The goal of the study was to analyze our experience in the control of hemorrhage in major liver trauma.

\section{Materials and Methods}

Our study was performed prospectively and it comprised the period of time from January 2009 till December 2012. In our study we analyzed 173 patients (The study sample included 173 patients) with liver trauma undergoing treatment in the Trauma UHC, Tirana. Parenchymal and vascular liver injuries were assessed according to the Moore 
E.E. classification revised in 1994 by the AAST.

The Injury Severity Score (ISS) system was used to determine the anatomical gravity of the concomitant injuries. ISS values vary from 0-75.

The Revised Trauma Score (RTS) classification was used to asses potential mortality. RTS values vary in between 0 8.

Based on the required criteria for conservative treatment of a liver injury, patients were divided in two main groups: A, B.

Group A - the group of patients that fulfills the criteria for conservative treatment.

Group A1 - patients managed successfully through conservative treatment.

Group A2 - patients in which conservative treatment failed.

Group B - the group of patients undergoing urgent laparotomy.

Group B1 - patients undergoing laparotomy.

Group B2 - patients undergoing surgery divided in two sessions.

\section{Results}

Median age of patients was 23.4 (the age range was from 6 to 75 years old).Main causes of liver trauma were as follows: motor vehicle accidents (including pedestrian accidents?) 88 patients (50.9 $\%)$, falls from a height 32 patients $(18.4 \%)$, gunshot wounds 24 patients (13.8\%), stab wounds 19 patients (11\%), direct blows 8 patients $(4.7 \%)$, iatrogenic hepatic injury 1 patient $(0.6 \%)$, electric injury from the electrical arc phenomena 1 patient $(0.6 \%)$.

Mechanism of liver trauma was as follows: Blunt trauma 129 patients (74.6\%). Open (/penetrating) trauma 44 patients $(25.4 \%)$. Penetrating trauma in gunshot wounds 25 patients (56.8\%). Penetrating trauma from stab wounds 19 patients $(43.2 \%)$.

Types of injuries according to Moore classification were as follows: liver rupture (81.\%), intraparenchymal hematoma $(9.2 \%)$, subcapsular hematoma ( $8 \%)$, injury to the hepatic segment (retro-hepatic) of inferior vena cava $(0.6 \%)$. Hepatic avulsion (0.6\%), combustion of liver $(0.6 \%)$.

$\begin{array}{ccc}\text { Organ } & \text { Blunt Penetrating Total Percentage } \\ \text { traum } & \text { trauma }\end{array}$

\begin{tabular}{l|llll}
\hline Spleen & 14 & 2 & 16 & 9.2 \\
Kidney & 9 & 3 & 12 & 6.9 \\
$\begin{array}{l}\text { Pancreas } \\
\text { Stomach }\end{array}$ & 2 & 2 & 4 & 2.3 \\
$\begin{array}{l}\text { Small bowel } \\
\text { duodenum }\end{array}$ & 3 & 7 & 10 & 5.8 \\
& 3 & 9 & 12 & 6.9 \\
\end{tabular}




\begin{tabular}{l|llll}
$\begin{array}{l}\text { Large bowel }+ \\
\text { rectum }\end{array}$ & 3 & 6 & 9 & 5.2 \\
$\begin{array}{l}\text { Diaphragm } \\
\text { Esophagus }\end{array}$ & 8 & 27 & 35 & 20.2 \\
Urinary bladder & 1 & 1 & 1 & 0.6 \\
Total & $\mathbf{4 3}$ & 0 & 2 & 1.2 \\
& & 57 & $\mathbf{1 0 0}$ & 58.3
\end{tabular}

Table 1: Concomitant abdominal injuries and their mechanism of injury.

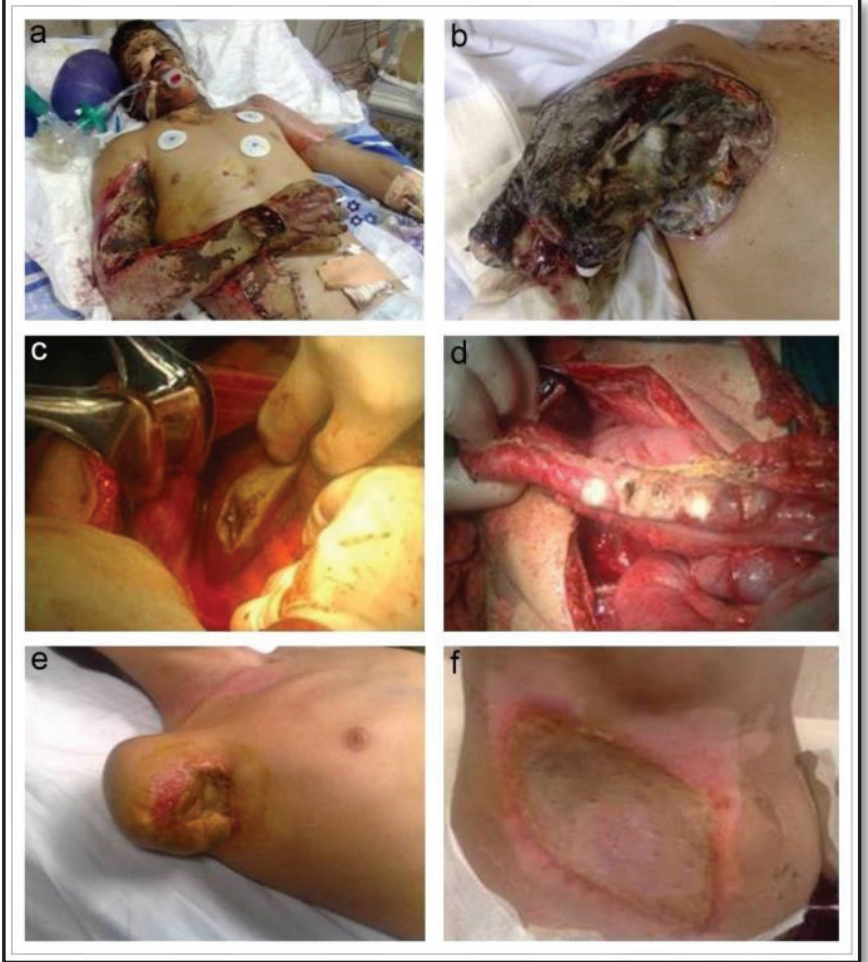

Figure 1: A.K., male, 16 years old, polytrauma patient due to electrical arc phenomena.

Injuries didn't occur as a result of direct contact to electrical circuit. Electrical fall/throw distance was $2-3 \mathrm{~cm}$ per $10.000 \mathrm{~V}$. Second grade combustion liver injury in the VIth segment. Rupture of the ascending and transverse colon. Large abdominal wall wound 20x15 cm with tissue loss and necrosis. Complex eventrated organs. Images of the evolution of the necrotic injuries in the right arm.

Liver injury distribution according to Couinaud segments were as follows: Segment no.I 2.5\%. Segment no.II 3.1\%. Segment III 5\%. Segmenti IVa 4\%. Segment IVb 6\%. Segment V 15.3\%. Segment VI 23.8\%. Segment VII 20.3\%. Segment VIII $20 \%$. 
Frequency of liver injuries according to MOORE grading were as follows: Ist grade injuries $(n=36) 20.8 \%$. Ind grade injuries $(n=60) 34,7 \%$. IIId grade injuries $(n=47) 27,2 \%$. IVth grade injuries $(n=21)$ $12,1 \%$. Vth grade injuries $(n=8) 4,6 \%$. VIth grade injuries $(n=1) 0,6 \%$.

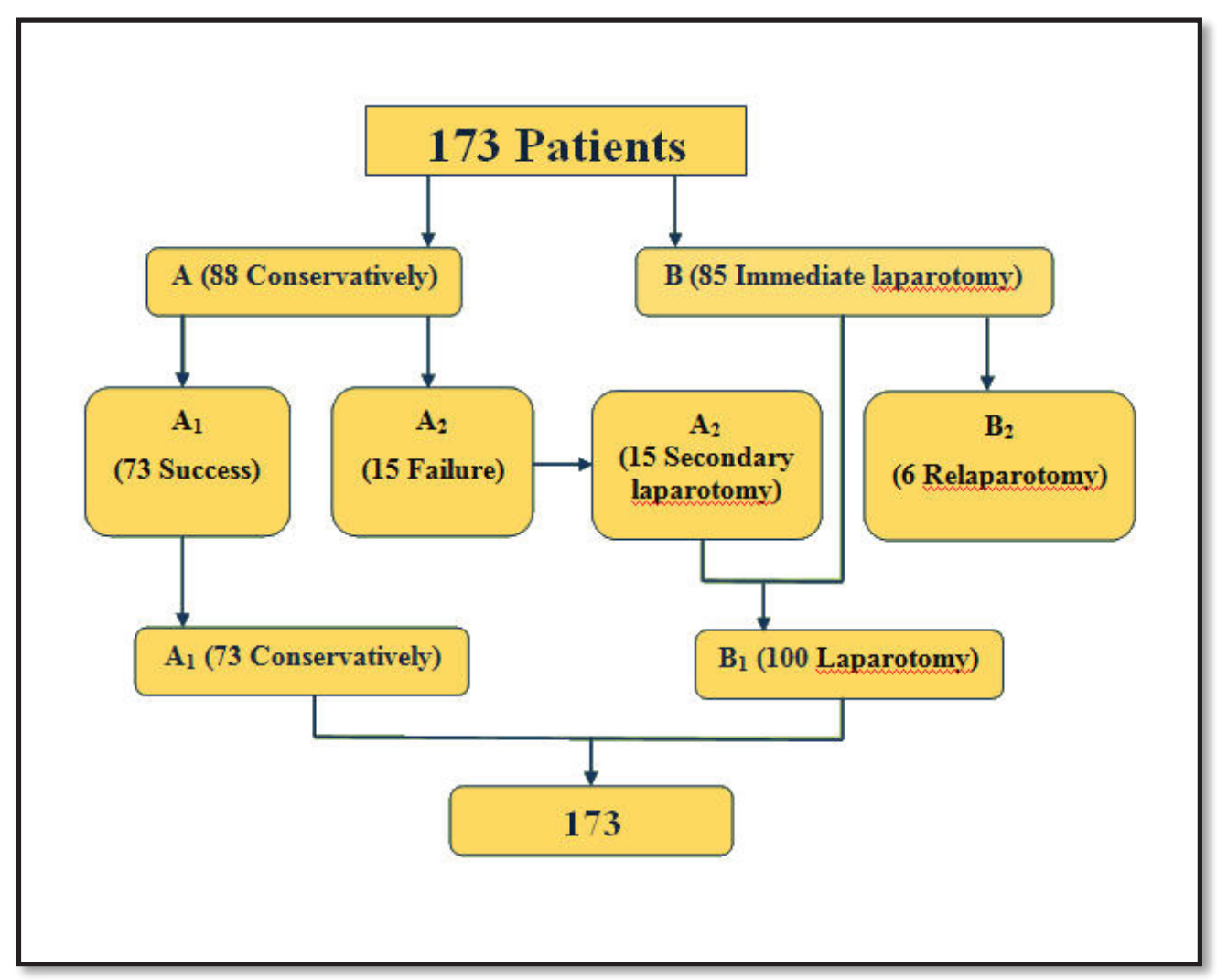

Figure 2: Initial determination of the treatment strategy in liver trauma and its evolution.

In our series of 173 patients, it was determined for group A with 88 patients $(50.9 \%)$ to initially undergo conservative treatment. It was determined for group B with 85 patients $(49.1 \%)$ to undergo urgent laparotomy. In group A1 conservative treatment was successful until patient discharge in 73 patients (42.2\%). Group A2 of 15 patients (17.2\%) consisted of patients with failed conservative treatment. Group B1 undergoing laparotomy included 100 patients (57.8\%). Group B2 consisted of 10 patients planned for surgery in two sessions. But for B2 group, we should emphasize that only 6 re-interventions with laparotomy were performed, because 4 patients didn't survive to undergo the 2 nd session of laparotomy.

\begin{tabular}{lll}
\hline Haemostasis maneuvers & Number of patients & Percentage \\
\hline Bimanual compression & 13 & 15.3 \\
\hline Pringle maneuver & 23 & 27
\end{tabular}




\begin{tabular}{lll}
\hline Perihepatic packing & 10 & 11.8 \\
\hline Foley catheter balloon tamponade & 2 & 2.4 \\
\hline Intrahepatic tamponage & 4 & 4.7 \\
\hline Total & 42 & 61.2 \\
\hline
\end{tabular}

Table 2: Temporary haemostasis maneuvers. Urgent laparotomy

Table 2 explains the temporary haemostasis maneuvers applied in cases when urgent laparotomy was indicated to control bleeding.

\section{Haemostasis maneuvers}

Electro-coagulation of the liver

injury

Haemostasis and

biliostasis

followed by hepatic closure

Haemostasis and

biliostasis

without hepatic closure

Pedicled omentum

hepatorrhaphy Anatomic liver

resection

Nonanatomic/atypical
Number of patients

12

41

6

7

1

7

1

75

\section{Percentage}

14.1

48.2

7.1

8.2

1.2

8.2

1.2

88

Table 3: Definitive haemostasis methods used in urgent laparotomy

Table 3 shows the surgical methods utilized in urgent laparotomies to obtain definitive haemostasis.

Perihepatic packing was performed in 10 patients, detailed as follows: It was indicated in 1 hemodynamically unstable polytrauma patient with IIId grade liver injury (\%). In 4 patients with
IVth grade liver injury $(40 \%)$, in 5 patients with Vth grade liver injury (50\%).

Left hepatectomy was performed in 1 patient. Right hepatectomy was performed in 1 patient.

Nonanatomic/atypical liver resections were performed in 4 cases. 


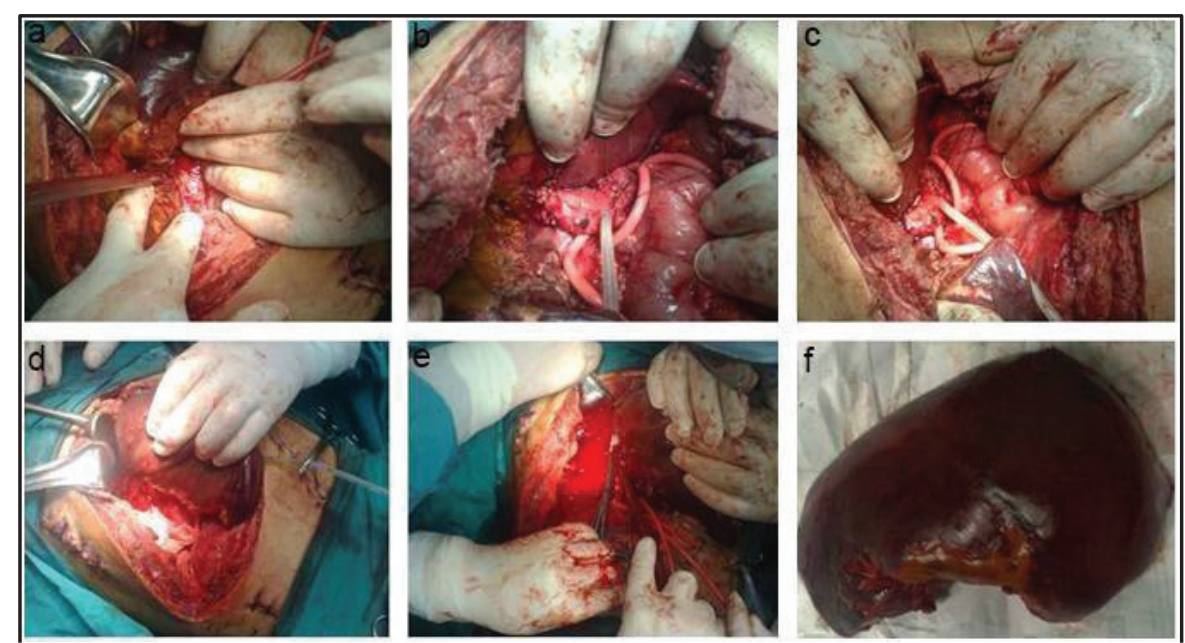

Figure 3: Images from the surgical intervention.

The preplanned second session of laparotomy, following the initial perihepatic packing. Stab wound (knife) penetrating liver injury. Vth grade liver injury of segments V and VIII. Injury to the hepatic segment of IVS. Suture of the hepatic segment of IVS. Right hepatectomy.

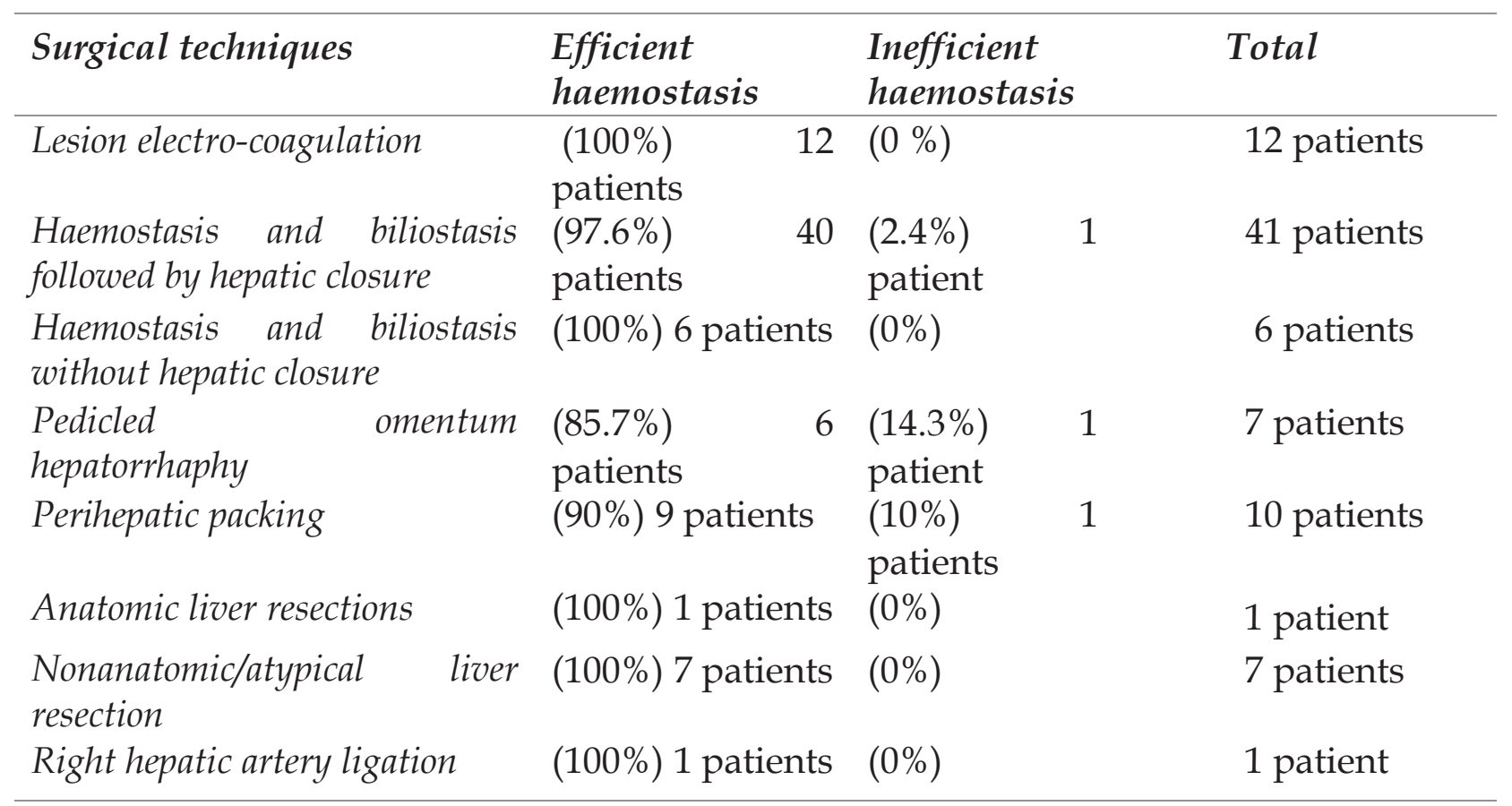

Table 4: Efficiency of the haemostasis maneuvers. 
Statistical analysis confirmed that the success of treatment of a liver injury with laparotomy was statistically significantly related to the grade of liver injury $(z=5.2912, p=<0.00001)$, other concomitant abdominal organ injuries $(z=4.0743, p=0.00005)$, amount of blood transfusion received $(p=0.03207)$, and age $(p=0.04944)$.

In isolated liver injuries, among statistically important factors in determining treatment success were complication rate $(p=0.01839)$, amount of blood transfused was correlated with the grade of liver injury $(z=2.2719$, $p=0.02309)$, cause of injury $(p=0.00062)$ and patients' age $(\mathrm{p}=0.08318)$.

Patients with ISS $\geq 20$ who underwent laparotomy: 83 patients $(83 \%)$. Patients with ISS $\leq 20$ who underwent laparotomy: 17 patients (17\%).

Patients with $\mathrm{RTS} \geq 7.8$ who underwent laparotomy: 35 patients (35\%), patients with RTS $\leq 7.8$ who underwent laparotomy: 65 patients (65\%).

\section{Discussion}

Surgical treatment of liver injury is closely associated with biological haemostasis correction and compensation of blood loss. Infusion of crystalloid fluids and fresh frozen plasma plays a crucial role in patient resuscitation in liver trauma. Administration of vasopressors os indicated in cases of vasoplegic shock.

Haemodinamic status of the patient remains a priority as it dictates either a conservative or surgical patient treatment. Intra-abdominal injuries may often be a cause of haemodynamic

instability, if during physical examination signs of peritonitis, penetrating wounds or abdominal distension are noticed. In further evaluating the above cases, rapid diagnostic modalities are indicated such as FAST ultrasound (focused assessment with sonography in trauma) and diagnostic peritoneal lavage (DLP). Utilization of computed tomography (CT) was the main diagnostic method of liver injuries in hemodynamically stable patients or patients hemodynamically stabilized following fluid resuscitation.

Nearly $85 \%$ of patients with blunt liver injury are reported to be hemodynamically stable or are hemodynamically stabilized following following fluid infusions (9). We found that around $87 \%$ of patients were hemodynamically stable or were hemodynamically stabilized following following fluid infusions.

A midline abdominal incision was the method of choice in performing laparotomy, but in a few cases, it is combined with transverse incision in the right upper abdominal quadrant (RUQ).

Intra-abdominal damage repair/control is obtained in a temporal or definitive way, depending on the hemodynamic status of the trauma patient. In hemodynamically stable patient with 
bleeding under control, all injuries are repaired in one intervention.

While, in hemodynamically unstable patient or patients with uncontrolled hemorrhage, injury repair is obtained through surgical interventions performed in two sessions.

The first action to be taken when a liver injury with active hemorrhage is evidenced is bimanual liver compression or compression of the organ against the diaphragm. Following this temporary hemorrhage control measure, the Pringle maneuver should be routinely performed (10). We have noticed that this maneuver is performed in a high percentage of our cases.

Following the Pringle maneuver, liver mobilization through ligament dissection was a necessity to ensure more access to the injured liver segments for damage control.

In minor liver injuries, hemorrhage control is obtained initially through packing of the bleeding site. Than inspection of the peritoneal cavity for other injuries is performed.

Haemostasis in liver injuries is obtained definitively or temporarily.

Haemostasis of decapsulated liver surfaces is obtained through electrocoagulation, argon beam coagulation or with absorbable (vicryl) haemostasis pads/mesh.

For injuries less than $3 \mathrm{~cm}$ deep, hepatorrhaphy has been the treatment of choice. In liver trauma accompanied with bleeding, exploration of the liver injury through opening its margins is performed, but only following the Pringle maneuver.
Pedicled omental hepatorrhaphy has resulted successful in liver injuries with concomitant venous injuries; only in one case it resulted to be non efficient.

This method was first used in 1975 by Stone. Later, Stone and Fabian reported to have achieved venous hemorrhage cessation with this method in $95 \%$ of patients with a mortality rate of $8 \%$ (11). Foley balloon catheter tamponade was another useful surgical maneuver in penetrating liver trauma. It was used in two patients with gunshot liver injury. Severe trauma cases with hemorrhage uncontrolled through other procedures, were managed through perihepatic packing.

This method has been initially used by Burckhardt in 1887, but it is successfully used nowadays, as well. According to Caruso et al., the decision for perihepatic packing should be taken immediately after exploration of the injury, to ensure the best chance for achieving patient survival (12). Richardson et al. have reported a significant decrease in mortality rates after 1989 in major liver trauma cases using immediate packing after exploration of the injury. (13)

We noticed that perihepatic packing was efficient in the hemorrhage control of all patients undergoing this procedure.

Perihepatic packing was removed in 48 -

72 hours (in ... cases), but in 4 cases packing removal was not obtained as 4 patients died within the first 48 hours.

Caruso et al. advocate perihepatic 
packing removal in 36-72 hours. Packing removal withing 72 hours is generally accepted as the optimal time for its removal to minimize perihepatic sepsis.

According to Krige et al. packing longer than 72 hours, had a frequency of developing perihepatic sepsis in $83 \%$ of cases, while if removed earlier than the 3-day time frame had as low a chance of developing perihepatic sepsis as $27 \%$. (14)

Hepatic artery ligation might be a useful maneuver for hemorrhage control in major liver injuries. If a significant hemorrhage reduction is noticed after the Pringle maneuver, then ligation of hepatic artery should be taken in consideration.

Our results in relation to selective hepatic artery ligation as a method of treatment coincide with literature reports. Right hepatic artery ligation was performed in an IVth grade liver injury to segments VI, VII and VIII.

When portal vein remains open (not obstructed), chances of major liver malfunction following hepatic artery ligation is minimal according to Aaron et al. (15).

We have noticed that, anatomic liver resections have shown a 100\% efficiency in achieving haemostasis and biliostasis. Nonanatomic/atypical liver resections resulted in an efficiency of $100 \%$ in achieving haemostasis and $85 \%$ in achieving biliostasis. Bile leak through drain tubes were noticed for several days.

It has been reported that major lobar resections may be a necessity in $10 \%$ of cases with liver injuries, but more than half of these resections result in patient death (16).

Strong et al. suggests that best results following such resections are achieved when the resection is performed by surgeons trained in hepatobiliary trauma (17).

Atypical resections correspond to proceeding a hepatectomy initiated by the traumatic event itself. Atypical resections are considered to be "economical" in the sense that the resection affects only the already injured parenchyma. Actually, the choice of the surgical plane in an atypical liver resection constitutes kind of a consensus

(18) It is required that the surgical plane of removal be near an anatomical plane to ease the identification and conserving of segmental pedicles, with the goal to achieve a "most economical/lvier conserving" resection.

In our observation of our series, only one patient underwent surgery due to liver compartment syndrome. Subcapsular hematomas should undergo surgery only for two reasons: 1- highly elevated levels of ALAT and ASAT, 2 - Budd-Chiari syndrome onset according to Markert DJ et al. and Letoublon C et al. (19.20).

Grade of liver injury was one of the statistically important factors in determining survival rates. Grade of liver injury resulted (-2.6), which means that for every single increase in the grade of injury chances of survival are reduced by (2.6).

Conservative management has failed in $17.1 \%$ of cases. In cases of 
concomitant intra-abdominal-injuries it was $2.3 \%$. The incidence (percentage) of failure of conservative treatment in cases with concomitant intra-abdominal injures is reported in between 0.5 to $3.5 \%$. (21.22).

Overall mortality was nearly $13 \%$ and it was closely related to hemorrhage shock - $42.9 \%$.

\section{Conclusions}

Perihepatic tamponage and performing the surgical intervention in two sessions has significantly /considerably increased/improved survival rates in major liver trauma. Treatment success in liver trauma is importantly correlated with the grade of liver injury, other concomitant intra-abdominal organ injuries, amount of blood transfused and age.

\section{References}

1. Feliciano D, Pachter H. Hepatic trauma revisited. Curr Prob Surg 1989; 28 : 453-524.

2. Jurkovich GJ, Hoyt DB, Moore FA, et al: Portal triad injuries . A multicenter study . J Trauma 39:426, 1995.

3. Dawson DL, Johansen $\mathrm{KH}$, Jurkovich GJ: Injuries to the portal triad. Am J Surg161:545, 1991.

4. Mirvis S, Whitley N, Vainwright $\mathrm{J}$, et al, Blunt hepatic trauma in adults: CT-based classification and correlation with prognosis and treatment. Radiology 1989; 171 : 27-32.

5. Croce M, Fabian T, Menke P, et al. Nonoperative management of blunt hepatic trauma is the treatment of choice for hemodynamically stable patients. Results of a prospective trial. Ann Surg 1995; 221 : 744-55.

6. Duane T, Como J, Bochiccchio G et al. Reevaluating the management and outcomes of severe blunt liver injury, J. Trauma $2004,57: 494$-500.

7. Pruvot F, Meaux F, Truant S, et al. Traumatismes graves fermés du foie: à la recherche de critères décisionnels pour le choix du traitement non opératoire. À propos d'une série de 88 cas. Ann Chir 2005; 130 : 70-80.

8. Velmahos G, Toutouzas $K$, and Radin $R$, et al. High success with nonoperative management of blunt hepatic trauma: the liver is a sturdy organ. Arch Surg 2003; 138 : 475-81.

9. Moore EE, Feliciano DV,Mattox KL: Trauma, 2004; 159-173.

10. Denis Castaing, Rene' Adam, Daniel Azoulay. Chirurgie du foie et de l'hypertension portale 2006;19: 164-67.

11. Stone HH ,Lamb JM: Use of pedicled omentum as an autogenous pack for control of hemorrhage in major injuries of the liver. Surg gyn Obstet 141:92,1975.

12. Caruso SM, Battistella FD, Owings JT, et al:Perihepatic 
packing of major liver injuries. Arch Surg 134:958, 1999.

13. Richardson JD, Franklin GA, Lukan JK, et al: Evolution in the management of hepatic trauma: A 25-year perspective. Ann Surg 232:324, 2000.

14. Krige JE, Bornman PC, Terblanche J: Therapeutic perihepatic packing in complex liver trauma. Br J surg 79:43, 1992.

15. Aaron Ws, Fulton RL, Mays ET: Selektive ligation of the hepatic artery for trauma of the liver. Surg Gynecol Obstet 141:187, 1975. 38 MooreEE, Feliciano DV,Mattox KL: Trauma, 2004; 159-173.

16. Beal SL: Fatal hepatic hemorrhage: An unresolved problem in the management of complex liver injuries. J Trauma 30:163, 1990.

17. Strong RS, Lynch SV, Wall DR,et al: Anatomic rezexion for sever liver trauma.Surgery 123:251, 1998.

18. Castaing D, Azoulay D, Adam R:Manoeuvres spe'ciales au cours des traumatismes du foie.Chirurgie $\mathrm{du}$ foie et de l'hypertension portale, 19:168170, 2006.

19. Markert DJ, Shanmuganathan K, Mirvis SE,et al. Budd-Chiari syndrome resulting from intrahepatic IVC compression secondary to blunt hepatic trauma. Clin Radiol 1997; 52: 384-7.

20. Letoublon C, Chen Y, Arvieux C, et al. Delayed Celiotomy or Laparoscopy as part of the Nonoperative Management of Blunt Hepatic Trauma. World J Surg 2008; 32:1189-93.

21. Pachter H, Knudson M, Esri B, et al. Status of nonoperative management of blunt hepatic injuries in 1995: a multicenter experience with 404 patients .J Trauma 1996; 40: 31-8.

22. Miller PR, Croce MA, Bee TK, Malhotra AK, Fabian

TC.Associated injuries in blunt solid organ trauma: implications for missed ingury in nonoperative management. J Trauma. 2002;53: 238-42. 